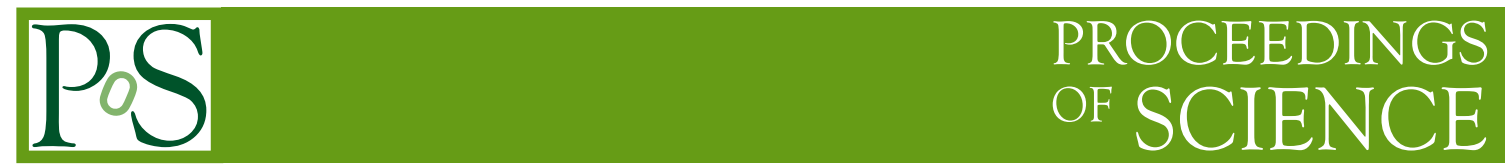

\title{
Hadronic Interactions from Lattice QCD
}

\section{André Walker-Loud*t \\ College of William and Mary \\ E-mail: walkloudewm.edu}

I will discuss recent results of the NPLQCD Collaboration regarding the calculation of hadronic interactions with lattice QCD. A particular emphasis will be spent on pi-pi scattering and other meson interactions.

8th Conference Quark Confinement and the Hadron Spectrum September 1-6, 2008

Mainz. Germany

*Speaker.

$\dagger$ I would like to thank my NPLQCD Collaborators who produced the results I am presenting. 


\section{Two-Particle Energy Levels in a Finite Euclidean Volume}

In a finite Euclidean volume, one does not calculate scattering matrix elements directly [1], but rather one calculates the volume dependent distortion of the two-particle energy levels due to the particle interactions which, below inelastic thresholds, can be related to the infinite volume scattering phase shift; this is known as Lüscher's method [2]. For example, for two degenerate particles in a cubic spatial volume of size $L^{3}$ and periodic boundary conditions, the distortion of the free-particle energy eigenvalues is related to the scattering phase shift through through a regulated three-dimensional summation,

$$
\Delta E_{2 m}=2 \sqrt{p^{2}+m^{2}}-2 m^{2} \quad \longrightarrow \quad \frac{p \cot \delta(p)}{m}=\frac{1}{m \pi L} S\left(\frac{p^{2} L^{2}}{16 \pi^{2}}\right)
$$

where $S(z)$ can be found in Refs. [3],

$$
S(z) \equiv \sum_{\mathbf{n}}^{|\mathbf{n}|<\Lambda} \frac{1}{|\mathbf{n}|^{2}-z}-4 \pi \Lambda
$$

\section{Precision $I=2 \pi \pi$ Scattering}

Low energy $\pi \pi$ scattering is theoretically the best understood hadron-hadron interaction. At leading order in chiral perturbation theory $(\chi \mathrm{PT})[4,5]$, the effective field theory describing the low-energy interactions of QCD, the s-wave scattering lengths are uniquely predicted [4]. The subleading corrections are known to one- [5] and two-loops [6]. Each of these higher order corrections depends upon unknown low-energy-constants (LECs) which must be determined from comparison with experiment or lattice calculations.

Low energy $\pi \pi$ interactions are also numerically very tractable as the pions are the lightest hadrons of QCD. The exotic $I=2 \pi \pi$ scattering channel ${ }^{1}$ is therefore an ideal process to study with lattice QCD before exploring the more challenging pion-nucleon and nucleon-nucleon interactions. The NPLQCD Collaboration performed the first lattice calculation of the $I=2 \pi \pi$ scattering length with $2+1$ dynamical flavors of light quarks (up, down, and strange) [7]. Using continuum $\chi$ PT to perform the extrapolation to the charged pion mass, they found $m_{\pi} a_{\pi \pi}^{I=2}=-0.0426 \pm 0.0019$. The calculation was performed by computing domain-wall valence quark propagators [8] on the Asqtad improved [9] coarse MILC lattices [10], the mixed-action lattice scheme of the LHP Collaboration [11]. Subsequently, a high-precision update of this result has been published [12], finding at the charged pion mass

$$
m_{\pi^{+}} a_{\pi \pi}^{I=2}=-0.04330 \pm 0.00042 .
$$

There are several ingredients which were necessary for this precision calculation; high statistics with the lattice calculation, systematic treatment of the lattice-artefacts from the discretization and finite volume, and chiral symmetry. A series of papers allowed for all of these systematic errors to be addressed in the updated calculation [12]. First, Ref. [12] included an order of magnitude increase in statistics compared to the original calculation [7], see Table 1 for details. In Refs. [13], the lattice spacing corrections to the scattering length were determined by utilizing the relevant

\footnotetext{
${ }^{1}$ Due to the disconnected quark diagrams, the scalar $I=0$ channel is numerically more expensive and noisier.
} 
Table 1: Parameters of NPLQCD lattice calculation of $I=2 \pi \pi$ scattering [12], using coarse MILC ensembles [10] with lattice spacing $b \sim 0.125 \mathrm{fm}$ and volume $L \sim 2.5 \mathrm{fm}$.

\begin{tabular}{c|cccc}
\hline \hline$\left\{m_{\pi}, m_{K}\right\}[\mathrm{MeV}]$ & $\{290,580\}$ & $\{350,595\}$ & $\{490,640\}$ & $\{590,675\}$ \\
$N_{c f g} \times N_{\text {source }}$ & $468 \times 16$ & $658 \times 20$ & $486 \times 24$ & $564 \times 8$ \\
\hline \hline
\end{tabular}

mixed-action $\chi$ PT. In Refs. [14], the exponentially suppressed volume corrections to Eq. (1.1) were determined. ${ }^{2}$ The other important ingredient is chiral symmetry, both the chiral symmetry of the domain-wall valence propagators used in the computation, as well as the fact that the $\pi \pi$ scattering lengths must vanish in the chiral limit. When expressed in terms of the lattice-physical pion mass and decay constant, the mixed-action extrapolation formula for the scattering length is given at one loop by [13]

$$
m_{\pi} a_{\pi \pi}^{I=2}=-(2 \pi) \frac{m_{\pi}^{2}}{\left(4 \pi f_{\pi}\right)^{2}}\left\{1+\frac{m_{\pi}^{2}}{\left(4 \pi f_{\pi}\right)^{2}}\left[3 \ln \left(\frac{m_{\pi}^{2}}{\mu^{2}}\right)-1-l_{\pi \pi}^{I=2}(\mu)-\frac{\tilde{\Delta}_{P Q}^{4}}{6 m_{\pi}^{4}}\right]\right\},
$$

where $l_{\pi \pi}^{I=2}(\mu)$ is the same linear combination of renormalized Gasser-Leutwyler coefficients as appears in continuum $\chi$ PT. There is no explicit dependence upon the valence-sea mesons [16] and the only explicit sea quark effects are contained in the last term, where for the quark mass tuning employed in Refs. [7, 12], $\tilde{\Delta}_{P Q}^{2}=b^{2} \Delta_{\mathrm{I}}$, which is the staggered taste-Identity splitting, which has been computed on the coarse MILC lattices [17]. The resulting chiral extrapolation is presented in Fig. 1. One striking feature of this lattice calculation, for the full range of quark masses used, the resulting scattering length is consistent Weinberg's tree level prediction [4]. Aside from the quantum corrections to the pion mass and decay constant, there is no evidence of higher order effects. This feature is not specific to $I=2 \pi \pi$ scattering but has also been found in computations of both the $I=3 / 2 K \pi$ and $I=1 K K$ scattering lengths [18].

\section{Nuclear and Hyper-Nuclear Interactions}

The main objective of the NPLQCD Collaboration is to make predictions for the structure and

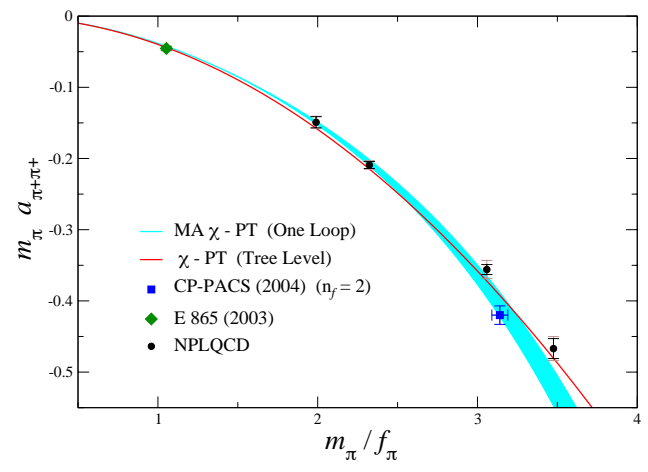

Figure 1: Chiral extrapolation of $I=2 \pi \pi$ scattering length using mixed-action $\chi P T$.

\footnotetext{
${ }^{2}$ The volume corrections computed in Ref. [14] were determined with continuum $\chi$ PT. The volume corrections for partially-quenched/mixed-action theories are also known [15] and were used in Ref. [12].
} 

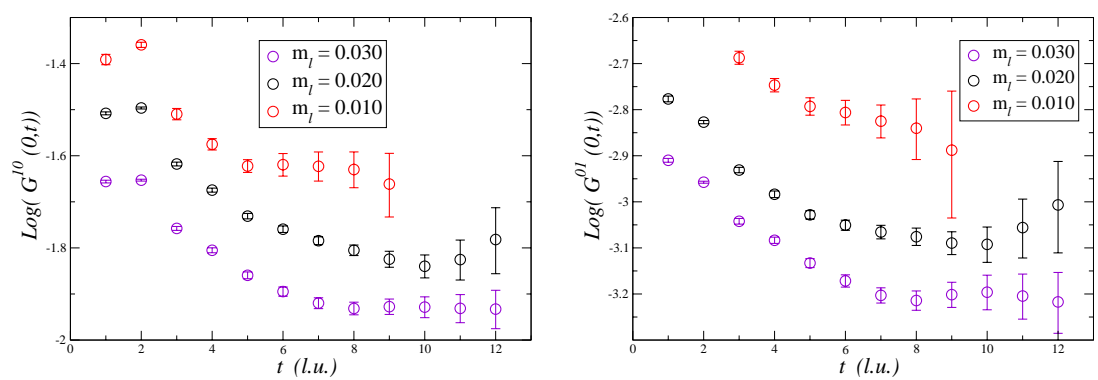

Figure 2: ${ }^{1} S_{0}$ and ${ }^{3} S_{1}$ nucleon-nucleon correlation functions taken from Ref. [19].

interactions of nuclei using lattice QCD. To that end, the first dynamical calculations of nucleonnucleon and hyperon-nucleon scattering lengths were performed recently [19]. However, lattice calculations of (multi)-baryon correlation functions suffer from a exponential degradation of the signal-to-noise [20], as can be seen in Fig. 2. This noise degradation is currently the main obstacle to computing multi-baryon correlation functions at light pion masses. Solutions to this problem will require several orders of magnitude increase in statistics as compared to the calculations in Refs. [19], especially as one utilizes lighter quark masses. There has been a proposal to alleviate this noise problem with a clever choice of boundary conditions [21], although this proposal has yet to be implemented numerically.

\section{Kaon Condensation}

In addition to calculating properties of two pions or two nucleons, the NPLQCD Collaboration has utilized these same computing resources to calculate properties of up to twelve pions and kaons $[22,23]$. In order to analyze these correlation functions, the energy levels of $n$ non-relativistic identical bosons interacting in a periodic cubic spatial volume of length $L$ was determined through $\mathscr{O}\left(L^{-7}\right)$ [24]. For example, we were able to compute the kaon chemical potential as a function the density of $\mathrm{K}^{-} \mathrm{s}$ at densities relevant for neutron star phenomenology. Additionally, three-pion and three-kaon interactions were determined from these calculations.

\section{Conclusions}

The use of lattice field theory methods to compute properties of hadron interactions has rapidly progressed in the last few years. Along the path to computing two-baryon interactions, we have seen a precision calculation of the $I=2 \pi \pi$ scattering length as well as the computation of systems containing up to 12 pions or kaons, allowing the first prediction for both the three-pion and threekaon interactions. In the next few years we will continue to see this rapid progression as the lattice community moves into the era of peta-flop computations.

\section{References}

[1] L. Maiani and M. Testa, Phys. Lett. B 245 (1990) 585.

[2] H. W. Hamber, E. Marinari, G. Parisi and C. Rebbi, Nucl. Phys. B 225 (1983) 475; M. Luscher, Commun. Math. Phys. 105 (1986) 153; M. Luscher, Nucl. Phys. B 354 (1991) 531. 
[3] S. R. Beane, P. F. Bedaque, A. Parreno and M. J. Savage, Nucl. Phys. A 747 (2005) 55; S. R. Beane, P. F. Bedaque, A. Parreno and M. J. Savage, Phys. Lett. B 585 (2004) 106.

[4] S. Weinberg, Phys. Rev. Lett. 17 (1966) 616.

[5] J. Gasser and H. Leutwyler, Annals Phys. 158 (1984) 142.

[6] J. Bijnens, G. Colangelo, G. Ecker, J. Gasser and M. E. Sainio, Phys. Lett. B 374 (1996) 210 [arXiv:hep-ph/9511397]; J. Bijnens, G. Colangelo, G. Ecker, J. Gasser and M. E. Sainio, Nucl. Phys. B 508 (1997) 263 [Erratum-ibid. B 517 (1998) 639] [arXiv:hep-ph/9707291].

[7] S. R. Beane, P. F. Bedaque, K. Orginos and M. J. Savage [NPLQCD Collaboration], Phys. Rev. D 73 (2006) 054503 [arXiv:hep-lat/0506013].

[8] D. B. Kaplan, Phys. Lett. B 288 (1992) 342 [hep-lat/9206013]; Y. Shamir, Nucl. Phys. B 406 (1993) 90 [hep-lat/9303005]; V. Furman and Y. Shamir, Nucl. Phys. B 439 (1995) 54 [hep-lat/9405004].

[9] K. Orginos, D. Toussaint and R. L. Sugar [MILC Collaboration], Phys. Rev. D 60 (1999) 054503; K. Orginos and D. Toussaint [MILC collaboration], Phys. Rev. D 59 (1999) 014501.

[10] C. W. Bernard et al., Phys. Rev. D 64 (2001) 054506 [arXiv:hep-lat/0104002].

[11] A. Walker-Loud et al., arXiv:0806.4549 [hep-lat].

[12] S. R. Beane, T. C. Luu, K. Orginos, A. Parreno, M. J. Savage, A. Torok and A. Walker-Loud, Phys. Rev. D 77, 014505 (2008) [arXiv:0706.3026 [hep-lat]].

[13] J. W. Chen, D. O’Connell, R. S. Van de Water and A. Walker-Loud, Phys. Rev. D 73 (2006) 074510; J. W. Chen, D. O'Connell and A. Walker-Loud, Phys. Rev. D 75 (2007) 054501; J. W. Chen, D. O'Connell and A. Walker-Loud, arXiv:0706.0035 [hep-lat].

[14] P. F. Bedaque, I. Sato and A. Walker-Loud, Phys. Rev. D 73 (2006) 074501 [arXiv:hep-lat/0601033].

[15] A. Walker-Loud, in preparation.

[16] K. Orginos and A. Walker-Loud, Phys. Rev. D 77 (2008) 094505 [arXiv:0705.0572 [hep-lat]].

[17] C. Aubin et al. [MILC Collaboration], Phys. Rev. D 70 (2004) 114501 [arXiv:hep-lat/0407028].

[18] S. R. Beane, P. F. Bedaque, T. C. Luu, K. Orginos, E. Pallante, A. Parreno and M. J. Savage, Phys. Rev. D 74 (2006) 114503; S. R. Beane, T. C. Luu, K. Orginos, A. Parreno, M. J. Savage, A. Torok and A. Walker-Loud [NPLQCD Collaboration], Phys. Rev. D 77 (2008) 094507.

[19] S. R. Beane, P. F. Bedaque, K. Orginos and M. J. Savage, Phys. Rev. Lett. 97 (2006) 012001; S. R. Beane, P. F. Bedaque, T. C. Luu, K. Orginos, E. Pallante, A. Parreno and M. J. Savage Nucl. Phys. A 794 (2007) 62.

[20] G. P. Lepage, lectures given at TASI 89 Summer School, Boulder, CO (1989).

[21] P. F. Bedaque and A. Walker-Loud, Phys. Lett. B 660 (2008) 369 [arXiv:0708.0207 [hep-lat]]; P. F. Bedaque and A. Walker-Loud, arXiv:0811.2127 [hep-lat].

[22] S. R. Beane, W. Detmold, T. C. Luu, K. Orginos, M. J. Savage and A. Torok, Phys. Rev. Lett. 100 (2008) 082004 [arXiv:0710.1827 [hep-lat]]; W. Detmold, M. J. Savage, A. Torok, S. R. Beane, T. C. Luu, K. Orginos and A. Parreno, arXiv:0803.2728 [hep-lat].

[23] W. Detmold, K. Orginos, M. J. Savage and A. Walker-Loud, Phys. Rev. D 78 (2008) 054514.

[24] S. R. Beane, W. Detmold and M. J. Savage, Phys. Rev. D 76, 074507 (2007) [arXiv:0707.1670]; W. Detmold and M. J. Savage, Phys. Rev. D 77, 057502 (2008) [arXiv:0801.0763 [hep-lat]]. 